\title{
BMJ Open Maternal lifestyle and nutritional status in relation to pregnancy and infant health outcomes in Western China: protocol for a prospective cohort study
}

\author{
Li Tang, ${ }^{1}$ Xiong-Fei Pan, ${ }^{1,2}$ Andy H Lee, ${ }^{3}$ Colin W Binns, ${ }^{3}$ Chun-Xia Yang, ${ }^{4}$ Xin Sun ${ }^{1}$
}

To cite: Tang L, Pan X-F, Lee AH, et al. Maternal lifestyle and nutritional status in relation to pregnancy and infant health outcomes in Western China: protocol for a prospective cohort study. BMJ Open 2017;7:e014874. doi:10.1136/ bmjopen-2016-014874

- Prepublication history for this paper is available online. To view these files please visit the journal online (http://dx.doi org/10.1136/bmjopen-2016014874).

LT and X-FP contributed equally.

Received 24 October 2016 Revised 17 February 2017 Accepted 21 March 2017

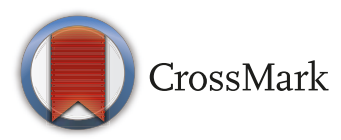

${ }^{1}$ Chinese Evidence-based Medicine Center, West China Hospital, Sichuan University, Chengdu, China

${ }^{2}$ Department of Epidemiology and Biostatistics, School of Public Health, Tongji Medical College, Huazhong University of Science and Technology, Wuhan, China

${ }^{3}$ School of Public Health, Curtin University, Perth, WA, Australia ${ }^{4}$ Department of Epidemiology and Biostatistics, West China School of Public Health, Sichuan University, Chengdu, China

Correspondence to

Dr Xin Sun;

sunx79@hotmail.com

\section{ABSTRACT}

Introduction Improving the health and nutrition of women and children is a priority for Western China, where the economy is less developed. Due to the dynamic nature of lifestyle, modern food habits and nutrition, there is a need to update our limited knowledge and understanding of maternal lifestyle and nutritional status and their impact on pregnancy and infant health outcomes. While breast milk is the preferred feeding option, infant formula use is widespread in China. It is thus necessary to examine the effects of formula consumption on growth and morbidity. Methods and analysis This is an ongoing prospective cohort study started in 2015 in Chengdu, Sichuan Province. A sample of 1901 pregnant women at 15-20 weeks of gestation were recruited from four maternal and child health hospitals and are followed prospectively to 12 months post partum. Detailed information on maternal lifestyle and nutritional status, obstetric complications, pregnancy outcomes, infant feeding practices, illnesses of the mother and infant and growth trajectory is collected through personal interviews, anthropometric measures and medical records and local health management system records retrieval. Multilevel mixed regression models, adjusted for clustering, will be applied to investigate the association between various exposure variables of interest and the longitudinal outcomes, taking into account the correlated data structure and the nesting of observations. Kaplan-Meier test and Cox regression analysis will be used to analyse the time-to-event data.

Ethics and dissemination Ethical approval has been obtained from the ethics committee of West China School of Public Health, Sichuan University and the Human Research Ethics Committee of Curtin University. Results will be presented at national and international conferences and published in peer-reviewed journals.

\section{INTRODUCTION}

\section{Background}

Despite substantial achievements in maternal and child health over the past two decades, China now faces a double burden of malnutrition, which is the persistence of undernutrition, along with a rapid rise in overweight and obesity in both children and mothers. A national survey showed that the proportion of undernutrition among

\section{Strengths and limitations of this study}

This is the first multicentre, prospective cohort study to investigate maternal dietary intake and physical activity during pregnancy and their impact on pregnancy outcomes as well as maternal and infant health in Western China.

- All the questionnaires administered have been validated by previous research.

- Recall errors and bias in the dietary intake and physical activity assessments may not be ruled out.

- Although all interviewers were trained in the administration of questionnaires, misunderstanding may still occur due to the poor education level of some participants.

- Gestational age at recruitment was set at 15-20 weeks; therefore, some information such as maternal weight, physical activity and diet during the first trimester could not be obtained.

- Lost to follow-up is expected in view of the current internal migration in China.

- Data retrieved from medical records and the local health management system may be of variable quality and subject to incompleteness.

women of reproductive age were $8.1 \%$ and $7.5 \%$ in urban and rural areas, respectively. ${ }^{1}$ With rapid economic development, Chinese women experience changes in their lifestyle and dietary behaviours. According to the WHO's Global Information Database, the prevalence of overweight and obesity (body mass index $(\mathrm{BMI}) \geq 25.0 \mathrm{~kg} / \mathrm{m}^{2}$ ) in Chinese females aged $\geq 15$ years has increased markedly from $22.7 \%$ in 2002 to $32.0 \%$ in $2010 .^{2}$ At the same time, there is now a greater proportion of Chinese infants born too small or too large. The Unicef statistics report a low birth weight $(<2500 \mathrm{~g})$ rate of $2 \%$ in China; however, recent reports suggest that it is approximately $6 \%$ and the rate may be increasing. ${ }^{3}$ With increases in the prevalence of maternal obesity and diabetes, a rapid increase in the rate of macrosomia 
(birth weight $\geq 4000 \mathrm{~g}$ ) has also been reported in China. According to the Chinese national nutrition and health survey in 2002, the prevalence of macrosomia was estimated at 5.3\%. ${ }^{4}$ A more recent survey conducted in 2011 in 14 provinces of China revealed an overall prevalence of macrosomia of $7.3 \%$, ranging from $4.1 \%$ to $13.4 \%$ in different provinces. ${ }^{5}$

During pregnancy, it is essential that the mother's diet contains adequate nutrients and energy at each stage to allow proper fetal growth and to maintain her own health. ${ }^{6}$ Dietary deficiencies are associated with intrauterine growth retardation, premature birth $(<37$ weeks gestation), low and malformation such as neural tube defects. ${ }^{7}$ On the other hand, overnutrition in pregnancy has been linked to developing metabolic disorders such as gestational diabetes mellitus and obesity due to excessive weight gain, as well as health-related problems for the infants in their later life. ${ }^{89}$ In addition to adequate nutritional intake, all pregnant women are advised to engage in appropriate levels of physical activity throughout pregnancy to minimise detrimental health risks. At least 150 min of moderate intensity activity (classified as 3 to 5 metabolic equivalent tasks) per week is recommended for pregnant women without medical problems. ${ }^{10}$ However, Chinese women often become sedentary once they are pregnant. Many of them even stop doing housework due to the traditional belief that activities such as sweeping and moving furniture, may lead to miscarriage or premature birth. Low levels of physical activity are known to result in excessive gestational weight gain, a major contributor to postpartum weight retention and long-term overweight and obesity. ${ }^{11}$

After birth, appropriate feeding practices play a crucial role in preventing mortality and achieving optimal growth, development and health outcomes. Both the WHO and the Chinese Ministry of Health recommend that infants should be exclusively breastfed for the first 6 months of life and continued breastfeeding thereafter with appropriate complementary foods. ${ }^{12}{ }^{13}$ However, the exclusive breastfeeding rate remains low whereas infant formula is widely consumed across China, either as a first feed or for complementary feeding. A recent prospective study conducted in Shihezi, Xinjiang Uygur Autonomous Region showed that less than $3 \%$ of infants were exclusively breastfed, for the first 6 months, when measured prospectively using a strict definition. ${ }^{14}$ In Zhejiang Province, over half the infants had already consumed, or were consuming, some infant formula by 1 month post partum and the proportion reached $98 \%$ by 6 months. ${ }^{15}$ What is of concern, in rural Sichuan Province, $68 \%$ of newborn infants received infant formula as their first feed. ${ }^{16}$ The infant feeding practices are far below the recommended target, with high rates of infant formula usage reported in both urban and rural areas. ${ }^{1517}$

To date, information is still lacking on the lifestyle and dietary intake of pregnant women in Western China, their habitual level of physical activity and their impacts on pregnancy and infant health outcomes. Western China is economically less developed than the Eastern coastal areas of China. There are still wide differences in maternal and infant health between the eastern and western regions. For instance, the maternal mortality ratio (MMR) and infant mortality rate (IMR) in Zhejiang Province, Eastern China, were respectively 5.28 per 100000 live births and 3.27 per 1000 live births in $2015,{ }^{18}$ similar to developed countries. However, in Sichuan Province, Western China, the MMR and IMR in 2015 remained high at 21.68 per 100000 live births and 7.80 per 1000 live births, respectively. ${ }^{19}$ Great efforts are required to improve the maternal and infant health in Western China.

Importantly, the prevalence of overweight and obesity is increasing rapidly worldwide, including China. ${ }^{20}$ Excessive weight, especially obesity, is known to be the major cause of morbidity and shortened life expectancy and represent a heavy burden on the healthcare system. $^{21}{ }^{22}$ In recent decades, the Developmental Origins of Health and Disease hypothesis has emerged to understand the origins of obesity and chronic disease. It highlights the link between the periconceptual, fetal and early infant phases of life and the subsequent development of adult obesity and related health disorders. ${ }^{23}{ }^{24}$ Both infants with a high birth weight and those with a low birth weight, followed by growth acceleration during infancy, have an increased risk of obesity in later life. ${ }^{25} 26$

\section{Aim and objectives}

The overall aim of the project is to identify modifiable maternal risk factors for adverse pregnancy and infant health outcomes in Western China.

The specific objectives include:

i. To assess the lifestyle, nutritional and metabolic status of pregnant women in Western China, including physical activity, smoking, alcohol drinking, dietary intake, maternal BMI, body fat composition and gestational weight gain.

ii. To investigate the consumption of infant formula (and other foods and beverages) by infants and ascertain their (its) effects on infant growth and morbidity within 12 months of birth.

iii. To ascertain the association between maternal and metabolic factors (eg, maternal BMI, dietary intake, physical activity and gestational diabetes mellitus) and adverse pregnancy outcomes (eg, preterm delivery, caesarean section, low birth weight, macrosomia, postpartum haemorrhage and admission to a neonatal intensive care unit).

iv. To determine (a) the prevalence of maternal overweight and obesity and its relationship with excessive gestational weight gain, postpartum bleeding and health outcomes; (b) the prevalence of maternal underweight status and its association with inadequate gestational weight gain and health outcomes. 
v. To evaluate the association between maternal BMI and lactation outcomes, including the intention, initiation, intensity and duration of breastfeeding.

vi. To investigate the consumption of infant formula and ascertain its effects on infant growth and morbidity within 12 months of birth.

\section{METHODS AND ANALYSIS}

\section{Study design and setting}

A multi-centre prospective cohort design is used. The study has started in May 2015 and is ongoing. Pregnant women at 15-20 weeks of gestation are recruited from four maternal and child health hospitals in Chengdu, Sichuan Province and followed until 12 months post partum. Gestational age at recruitment was set at 15-20 weeks since the healthcare booklet is available to pregnant women in China only at or after 12 weeks of gestation, and the majority of Chinese women attended their first antenatal visit after the first trimester. ${ }^{27}$ The follow-up time will be extended to 24 months if further funding is obtained. As the capital city of Sichuan Province, Chengdu is the economic centre of Western China with a gross domestic product of 83849 yuan $(\sim 12440)$ per capita in $2014 .^{28}$ The reported proportion of supervised delivery at hospital in Sichuan Province was 98.13\% in $2015 .^{19}$

\section{Sample size determination}

A sample size estimation formula for comparison of two proportions was used to calculate the sample size of this study as follows:

$$
n=\left[\frac{z_{\alpha / 2} \sqrt{\Pi(1-\Pi)\left(Q_{1}^{-1}+Q_{2}^{-1}\right)}+z_{\beta} \sqrt{\Pi_{1}\left(1-\Pi_{1}\right) Q_{1}^{-1}+\Pi_{2}\left(1-\Pi_{2}\right) Q_{2}^{-1}}}{\delta}\right]^{2}
$$

where $n$ is the required sample size; $Z_{\alpha / 2}$ and $Z_{\beta}$ are the $Z$ values corresponding to the alpha and beta error, respectively; $\varpi_{1}$ is the estimated proportion of macrosomia in overweight/obese women; $\varpi_{2}$ is the estimated proportion of macrosomia in normal/underweight women; $Q_{1}$ and $Q_{2}$ are the proportion of participants in each group; $\varpi=Q_{1} \varpi_{1}+Q_{2} \varpi_{2} ; \delta=\varpi_{1}-\varpi_{2}$.

According to a recent survey conducted in 14 provinces of China, the incidence of macrosomia at birth was $7.3 \% .^{5}$ In Western China, $30 \%$ of women are estimated to be overweight/obese (BMI $\geq 24 \mathrm{~kg} / \mathrm{m}^{2}$ ) before or during early pregnancy. The proportions of macrosomia in overweight/obese and normal/underweight women are projected at $10 \%$ and $6 \%$, respectively. With a statistical power of $80 \%$ and a significance level of $5 \%$, a sample of 1650 women are required to detect a $4 \%$ difference in macrosomia rates between the two groups at childbirth. To allow for $10 \%$ attrition and withdrawals during pregnancy and follow-up, a minimum sample of 1850 pregnant women at baseline was required.

\section{Recruitment}

All pregnant women who attended the study hospitals for their routine antenatal care at 15-20 weeks were approached and invited to participate in the study. A written information sheet was given to all potential participants before obtaining their informed consent. Recruitment of the study cohort started in May 2015 and was finished in August 2015. A total of 1901 women were recruited.

\section{Eligibility criteria}

Inclusion criteria were local residents aged 18-40 years with a singleton pregnancy. Women were excluded if they became pregnant after infertility treatment including in vitro fertilisation or intrauterine insemination, were suffering from serious chronic health conditions or severe infectious diseases such as HIV or were unable to answer the questions during the interview.

\section{Data collection}

After enrolment, participants who gave informed consent were interviewed face-to-face by trained nurses using a structured questionnaire to obtain information on demographic, socioeconomic and health characteristics, dietary intakes, as well as lifestyle including physical activity, cigarette smoking and alcohol drinking. Anthropometric, body fat composition and blood pressure measurements were also taken at the baseline interview. During their visit to the hospitals at 32-37 weeks of gestation, participants were administered a questionnaire to inquire on their attitudes towards infant feeding and antenatal depression symptoms.

Maternal weight at each antenatal visit and obstetric complications during pregnancy (eg, gestational diabetes, pregnancy-induced hypertension and pre-eclampsia) were extracted from medical records. At the time of birth, details including obstetric and neonatal outcomes (eg, type of delivery, appearance, pulse, grimace, activity, respiration (APGAR) scores, birth weight and problems/ complications during delivery) were recorded. Breastfeeding initiation practices and maternal breastfeeding confidence in early postpartum period were assessed by personal interview before hospital discharge.

The cohort is subsequently followed up by telephone interviews at 1, 3, 6 and 12 months postpartum to collect detailed information on infant feeding practices, infant illnesses, breastfeeding and other health problems of both mothers and infants. Data on infant growth (weight and length) between 1 and 12 months will be retrieved from the local Maternal and Child Health Management System on the last follow-up.

The follow-ups are still in progress and expected to be completed by April 2017. The recruitment and data collection procedure is presented in figure 1. 
All pregnant women attending study hospitals for their antenatal care at 15-20 weeks were identified and approached

- Screening for eligibility

- Obtaining informed consent

\section{Enrolment}

- Baseline face-to-face interview: demographic, socioeconomic and health characteristics, physical activity, dietary intake, alcohol drinking and smoking behaviours

- Anthropometric and body fat composition measurements

- Systolic and diastolic blood pressure measurements

\section{1st follow-up (32-37 weeks of gestation)}

- Face-to-face interview: attitudes towards infant feeding, antenatal depression symptoms

- Weight and body fat composition measurements

- Systolic and diastolic blood pressure measurements

\section{2nd follow-up (before hospital discharge)}

- Medical records: maternal weight at each antenatal visit, obstetric complications, neonatal and pregnancy outcomes

- Face-to-face interview: breastfeeding initiation practices, breastfeeding confidence

\section{Follow-ups at 1, 3, 6 and 12 months postpartum}

- Telephone interview: infant feeding practices, infant illnesses, maternal breastfeeding and health problems

- Data on infant growth to be retrieved from the local Maternal and Child Health Management System at 12 months postpartum

Figure 1. Flowchart of recruitment and data collection

Figure 1 Flow chart of recruitment and data collection.

\section{Variables and potential confounders}

The primary outcome variables of the study are gestational weight gain, birth weight and maternal and infant health problems. The secondary outcome variables include obstetric complications, mode of delivery, breastfeeding initiation and duration and infant growth at 1 year of age. Maternal BMI, physical activity and dietary intake were the main exposures of interest. The potential confounding variables were selected based on literature $^{51529}$ and include infant gender, gestational age at delivery, maternal age, education, socioeconomic status, parity, a history of diabetes and hypertension, alcohol drinking, maternal and paternal smoking during pregnancy, maternal breastfeeding confidence and depressive symptoms. The variables, the instrument used and the assessment time points are presented in table 1 .

\section{Measures}

Anthropometric measurements

A digital scale was used to record the weight of all participants to the nearest $100 \mathrm{~g}$. Height was measured using a stadiometer to $0.1 \mathrm{~cm}$. A bioelectrical impedance analysis scale was used to measure the body fat composition. Total gestational weight gain was estimated by subtracting the early first trimester weight from the last measured weight before delivery. Maternal BMI is calculated using 
Table 1 Variables, instruments used and assessment time points

\begin{tabular}{lll}
\hline Variables & Instruments & Assessment time points \\
\hline Primary outcomes & & Baseline; hospital discharge \\
$\begin{array}{l}\text { Gestational weight gain } \\
\text { Birth weight }\end{array}$ & Digital weight scale; medical records & Hospital discharge \\
$\begin{array}{l}\text { Maternal physical health problems after } \\
\text { childbirth }\end{array}$ & Medical records & $1,3,6$ and 12 months \\
Infant health problems & & postpartum \\
& Reported by the mother & $1,3,6$ and 12 months \\
\end{tabular}

Secondary outcomes

Obstetric complications during pregnancy and Medical records at birth

Type of delivery Medical records

Hospital discharge

Breastfeeding: prelacteal feeds, time to first Breastfeeding questionnaire

Hospital discharge

feed, initiation, duration

Infant growth at 1 year of age: infant weight

Medical records (infant weight and length at and length within 12 months

birth); the local Maternal and Child Health

Hospital discharge; 1, 3, 6 and 12 months postpartum

Management System (infant weight and

Hospital discharge; 12 months

length between 1 and 12 months)

\section{Exposure and confounding variables}

\begin{tabular}{|c|c|c|}
\hline Maternal weight and height at baseline & Digital weight scale; stadiometer & Baseline \\
\hline Body fat composition & Bioelectrical impedance analysis scale & Baseline \\
\hline Physical activity during pregnancy & Pregnancy physical activity questionnaire & Baseline \\
\hline $\begin{array}{l}\text { Dietary intake and alcohol drinking during } \\
\text { pregnancy }\end{array}$ & Food frequency questionnaire & Baseline \\
\hline $\begin{array}{l}\text { Demographic and socioeconomic } \\
\text { characteristics: age, marital status, education } \\
\text { level, occupation, household income }\end{array}$ & Structured questionnaire & Baseline \\
\hline $\begin{array}{l}\text { Health characteristics: history of diabetes and } \\
\text { hypertension, parity }\end{array}$ & Structured questionnaire & Baseline \\
\hline Infant gender, gestational age at delivery & Medical records & Hospital discharge \\
\hline Cigarette smoking & Structured questionnaire & $\begin{array}{l}\text { Baseline; } 6 \text { months } \\
\text { postpartum }\end{array}$ \\
\hline Maternal attitudes towards infant feeding & lowa Infant Feeding Attitude Scale & 32-37 weeks of gestation \\
\hline Maternal breastfeeding confidence & Breastfeeding Self-Efficacy Scale & Hospital discharge \\
\hline Maternal depressive symptoms & Edinburgh Postnatal Depression Scale & $\begin{array}{l}32-37 \text { weeks of gestation; } 1,3 \\
\text { and } 6 \text { months postpartum }\end{array}$ \\
\hline Infant dietary intake & 48 hours food recall & 12 months postpartum \\
\hline
\end{tabular}

weight and height at baseline $\left(\mathrm{kg} / \mathrm{m}^{2}\right)$. Participants with a BMI of $<18.5 \mathrm{~kg} / \mathrm{m}^{2}, 18.5-23.9 \mathrm{~kg} / \mathrm{m}^{2}, 24.0-27.9 \mathrm{~kg} / \mathrm{m}^{2}$ or $\geq 28 \mathrm{~kg} / \mathrm{m}^{2}$ are classified as underweight, normal, overweight or obese, respectively. ${ }^{30}$ Birth weight was measured on an electronic scale immediately after birth.

Physical activity measurements

The Pregnancy Physical Activity Questionnaire (PPAQ) measures the duration, frequency and intensity of physical activity during pregnancy. ${ }^{31}$ It has been translated and validated in different languages including Chinese. $^{32}$ The validated Chinese version of PPAQ was administered to solicit time spent participating in 31 activities, including household/caregiving (nine activities), occupational (five activities), sports/exercise (eight activities), transportation (four activities) and inactivity (five activities).

Dietary intake assessment

A validated and reliable Food Frequency Questionnaire developed for residents in Chongqing, Western China ${ }^{33}$ was used to determine the food consumption of participants at baseline. It consists of various food and beverage items grouped into categories, with frequency and quantities consumed recorded in detail. The frequency is either 'per day, per week, per month, per year or never' and a standard portion or utensil is defined for each food item listed. 


\section{Breastfeeding and infant diet assessment}

The breastfeeding questionnaires were taken from those used in previous cohort studies in China. ${ }^{34} 35$ Breastfeeding experience and problems encountered during the lactation period are also recorded. The WHO definitions for breastfeeding are adopted in this study. ${ }^{36}$ At 12 months postpartum, mothers will be asked to recall all foods and beverages consumed by their infants in the past 48 hours and to estimate the portion size of each item. Information will also be sought on the brands of infant formula used within the first year.

\section{Depressive symptoms assessment}

The presence of maternal depressive symptoms during pregnancy and postpartum period was assessed using the Edinburgh Postnatal Depression Scale (EPDS). ${ }^{37}$ EPDS has been widely used in antenatal and postnatal depression research. ${ }^{38}$ It consists of 10 items rated on a 4-point scale (from 0 to 3 ), with the total score ranging from 0 to 30 . It has been translated into Chinese for use in Western China. ${ }^{40}$

\section{Maternal attitudes towards infant feeding}

The Iowa Infant Feeding Attitude Scale (IIFAS) has been consistently reported to possess adequate reliability and validity in measuring maternal attitudes towards infant feeding in cross-cultural settings. ${ }^{41}$ The Chinese version of IIFAS was used in our study. ${ }^{42}$ It consists of 17 items with a 5-point Likert scale ranging from 1 (strongly disagree) to 5 (strongly agree). Approximately half the items are worded favourably towards breastfeeding and the remaining items favour formula feeding. Items favouring formula feeding are reverse-scored and a total score is computed by summing all items. Total attitude scores range from 17 to 85 with higher scores reflecting attitudes more positive to breastfeeding. Total scores are grouped into three categories: positive to breastfeeding (70-85); neutral (49-69); and positive to formula feeding (17-48).

\section{Maternal breastfeeding confidence measurement}

Maternal breastfeeding confidence was measured using the Breastfeeding Self-Efficacy Scale (BSES), which is a 33-item instrument. ${ }^{43}$ The items are preceded by the phrase 'I can always' and anchored with a 5-point Likert scale where $1=$ not at all confident and $5=$ always confident. All items are presented positively, and scores are summed to produce a range from 33 to 165. A higher score indicates a stronger confidence in breastfeeding. The Chinese version of BSES has been applied in previous research. $^{44}$

\section{Statistical analysis}

All data entry and statistical analyses will be performed in the SPSS package V.22. Summary statistics will be used to describe the profile of participants and to summarise the exposure and outcome variables. Group comparisons will be undertaken using $\chi^{2}$ tests for categorical characteristics, and either t-tests/analysis of variance or Mann-Whitney $\mathrm{U}$ tests for continuous variables.
In addition, multilevel mixed regression models, adjusted for clustering, will be applied to investigate the association between various exposure variables of interest and the longitudinal outcomes (eg, infant weight and length), taking into account the correlated data structure and the nesting of observations. Kaplan-Meier test and Cox regression analysis will be used to analyse the timeto-event data (eg, duration of breastfeeding). Univariate models will be initially performed to explore the association between exposure variables and each outcome. Interaction terms between independent variables will be examined to identify potential effect modification. Effects of potential confounders are adjusted in the multivariate mixed effects models. Crude and adjusted ORs and associated $95 \%$ CIs will be reported for logistic models, coefficients (95\% CIs) for linear regression models and HRs (95\% CIs) for Cox regression models.

\section{Ethics and dissemination}

The study protocol was approved by the Ethics Committee of West China School of Public Health, Sichuan University and the Human Research Ethics Committee of Curtin University. The participants were informed of the aims and nature of the research both verbally and via an information sheet. They were requested to complete and sign a consent form before enrolment. Confidentiality of the information provided by participants and their right to withdraw without prejudice are enforced throughout the study duration.

All individual data are securely stored, password protected and accessible by the research team only. The aggregated research findings will be presented at national and international scientific conferences and be submitted for publication in peer-reviewed journals. Furthermore, education seminars and training workshops will be held to share study findings with health professionals working with pregnant women and new mothers in Western China.

\section{DISCUSSION}

Pregnancy and the first year of life are critical periods for adult health. ${ }^{24}$ Due to the dynamic nature of lifestyle, modern food habits and nutrition, it is necessary to update our limited knowledge and understanding of maternal nutrition and lifestyle in Western China and their impact on pregnancy and infant health outcomes. Improvements in these areas will contribute towards increasing the quality of life and reducing morbidity and mortality, especially for those associated with overweight and obesity. Furthermore, assessment of breastfeeding and infant formula consumption on infant growth and health has implications towards enhancing the national infant feeding guidelines, especially in view of the widespread usage of infant formula and associated problems. ${ }^{15} 17$ Research findings from the multicentre prospective cohort study will provide an evidence base for developing effective maternal and child health strategies in Western China. 
Contributors AHL, CWB, CXY and XS conceived and designed the study. LT and XFP drafted the manuscript. AHL, CWB and XS critically revised the manuscript. All authors have read and approved the manuscript.

Funding This work is supported by China Medical Board under grant number 14-199.

Competing interests None declared.

Patient consent Obtained.

Ethics approval The ethics committee of West China School of Public Health, Sichuan University and the Human Research Ethics Committee of Curtin University.

Provenance and peer review Not commissioned; externally peer reviewed.

Open Access This is an Open Access article distributed in accordance with the Creative Commons Attribution Non Commercial (CC BY-NC 4.0) license, which permits others to distribute, remix, adapt, build upon this work non-commercially, and license their derivative works on different terms, provided the original work is properly cited and the use is non-commercial. See: http://creativecommons.org/ licenses/by-nc/4.0/

(C) Article author(s) (or their employer(s) unless otherwise stated in the text of the article) 2017. All rights reserved. No commercial use is permitted unless otherwise expressly granted.

\section{REFERENCES}

1. Lai J, Wang Y, Li N, et al. The nutrition and health status of reproductive age, pregnant and lactating women in China. Chin $J$ Prev Med 2009;43:154-8.

2. World Health Organization. Global infobase. Geneva: WHO, 2011. https://apps.who.int/infobase.

3. Chen Y, Li G, Ruan Y, et al. An epidemiological survey on low birth weight infants in China and analysis of outcomes of full-term low birth weight infants. BMC Pregnancy Childbirth 2013;13:242.

4. Yin S. The nutritional and health status of children aged 0-6 yearsChinese nutrition and health survey in 2002. Beijing: People's Medical Publishing House, 2008.

5. Li G, Kong L, Li Z, et al. Prevalence of macrosomia and its risk factors in China: a multicentre survey based on birth data involving 101,723 singleton term infants. Paediatr Perinat Epidemiol 2014;28:345-50.

6. Vause T, Martz P, Richard F, et al. Nutrition for healthy pregnancy outcomes. Appl Physiol Nutr Metab 2006;31:12-20.

7. Keen CL, Clegg MS, Hanna LA, et al. The plausibility of micronutrient deficiencies being a significant contributing factor to the occurrence of pregnancy complications. J Nutr 2003;133(5 Suppl 2):1597s-605.

8. Mehta SH. Nutrition and pregnancy. Clin Obstet Gynecol 2008;51:409-18.

9. Widen EM, Whyatt RM, Hoepner LA, et al. Excessive gestational weight gain is associated with long-term body fat and weight retention at 7 y postpartum in African American and dominican mothers with underweight, normal, and overweight prepregnancy BMI. Am J Clin Nutr 2015;102:1460-7.

10. Currie S, Sinclair M, Murphy MH, et al. Reducing the decline in physical activity during pregnancy: a systematic review of behaviour change interventions. PLoS One 2013;8:e66385.

11. Rong K, Yu K, Han X, et al. Pre-pregnancy BMI, gestational weight gain and postpartum weight retention: a meta-analysis of observational studies. Public Health Nutr 2015;18:2172-82.

12. World Health Organization and UNICEF, Global Strategy for Infant and Young Child Feeding. Geneva: WHO, 2003.

13. Health Ministry of China. [Infant and young child feeding strategy of China] Beijing: health Ministry of China; [updated 1 Aug 2007]. 2014 http://www.gov.cn/fwxx/jk/2007-08/01/content_703104.htm

14. Liu P, Qiao L, Xu F, et al. Factors associated with breastfeeding duration: a 30-month cohort study in northwest China. J Hum Lact 2013;29:253-9.

15. Qiu L, Binns CW, Zhao Y, et al. Breastfeeding practice in Zhejiang Province, PR China, in the context of melamine-contaminated formula milk. J Health Popul Nutr 2010;28:189-98.

16. Tang L, Binns CW, Lee AH, et al. Low prevalence of breastfeeding initiation within the first hour of life in a rural area of Sichuan Province, China. Birth 2013;40:134-42

17. Tang L, Binns CW, Lee AH. Infant formula crisis in China: a cohort study in sichuan province. J Health Popul Nutr 2015;33:117-22.

18. Zhejiang Provincial Bureau of Statistics, Survey Office of National Bureau of Statistics in Zhejiang. [Statistical bulletin of the national economic and social development of Zhejiang Province in 2015] Hangzhou: Zhejiang Provincial Bureau of Statistics; [updated 23 Feb 2016]. http://www.zj.stats.gov.cn/tjgb/gmjjshfzgb/201602/ t20160229_169661.html (accessed 18 Oct 2016).

19. Health and Family Planning Commission of Sichuan Province. [Statistical bulletin of health and family planning development of Sichuan Province in 2015] Chengdu: Health and Family Planning Commission of Sichuan Province; [updated 24 Mar 2016]. http:// www.scwst.gov.cn/index.php/zhdc/jbqk/12795-2015 (accessed 18 Oct 2016).

20. Wang R, Zhang P, Gao C, et al. Prevalence of overweight and obesity and some associated factors among adult residents of northeast China: a cross-sectional study. BMJ Open 2016;6:e010828.

21. Zhao W, Zhai Y, Hu J, et al. Economic burden of obesity-related chronic diseases in Mainland China. Obes Rev 2008;9:62-7.

22. Haslam DW, James WP. Obesity. Lancet 2005;366:1197-209.

23. Barker DJ. The developmental origins of well-being. Philos Trans $R$ Soc Lond B Biol Sci 2004;359:1359-66.

24. Barker DJ, Thornburg KL. The obstetric origins of health for a lifetime. Clin Obstet Gynecol 2013;56:511-9.

25. Ong KK, Loos RJ. Rapid infancy weight gain and subsequent obesity: systematic reviews and hopeful suggestions. Acta Paediatr 2006;95:904-8

26. Schellong K, Schulz S, Harder T, et al. Birth weight and long-term overweight risk: systematic review and a meta-analysis including 643,902 persons from 66 studies and 26 countries globally. PLoS One 2012;7:e47776.

27. Dou L, Wang X, Wang F, et al. Epidemic profile of maternal syphilis in China in 2013. Biomed Res Int 2016;2016:1-8.

28. Chengdu Bureau of Statstics. [Chengdu Statistical Yearbook 2015]. 25. Beijing: China Statistics Press, 2015.

29. Yusuff AS, Tang L, Binns CW, et al. Antenatal depressive symptoms and breastfeeding: a prospective cohort study. Breastfeed Med 2015;10:300-4.

30. Chinese center for Disease Control and Prevention. [Guidelines for the prevention and control of overweight and obesity in China]. Beijing: People's Medical Publishing House, 2006.

31. Chasan-Taber L, Schmidt MD, Roberts DE, et al. Development and validation of a Pregnancy Physical Activity Questionnaire. Med Sci Sports Exerc 2004;36:1750-60.

32. Zhang Y, Zhao Y, Dong S, et al. Reliability and validity of the Chinese version of the Pregnancy Physical Activity Questionnaire (PPAQ)] Chinese Journal of Nursing 2013;48:59-61.

33. Zhou ZY, Takezaki T, Mo BQ, et al. Development of a semiquantitative food frequency questionnaire to determine variation in nutrient intakes between urban and rural areas of Chongqing, China. Asia Pac J Clin Nutr 2004;13:273-83.

34. Qiu L, Zhao Y, Binns CW, et al. A cohort study of infant feeding practices in city, suburban and rural areas in Zhejiang Province, PR China. Int Breastfeed J 2008;3:4.

35. Xu F, Binns C, Zheng S, et al. Determinants of exclusive breastfeeding duration in Xinjiang, PR China. Asia Pac J Clin Nutr 2007:16:316-21.

36. World Health Organization. Indicators for assessing infant and young child feeding practices: conclusions of a consensus meeting held 6-8 November 2007 in Washington DC. 2008. Geneva: $\mathrm{WHO}: 4$

37. Cox JL, Holden JM, Sagovsky R. Detection of postnatal depression. development of the 10-item Edinburgh Postnatal Depression Scale. Br J Psychiatry 1987;150:782-6.

38. Khalifa DS, Glavin K, Bjertness E, et al. Determinants of postnatal depression in Sudanese women at 3 months postpartum: a crosssectional study. BMJ Open 2016;6:e009443.

39. Patton GC, Romaniuk H, Spry E, et al. Prediction of perinatal depression from adolescence and before conception (VIHCS): 20year prospective cohort study. Lancet 2015;386:875-83.

40. Wang Y, Guo X, Lau Y, et al. Psychometric evaluation of the Mainland Chinese version of the Edinburgh Postnatal Depression Scale. Int J Nurs Stud 2009;46:813-23.

41. Wallis $A B$, Brînzaniuc $A$, Chereche区 $R$, et al. Reliability and validity of the Romanian version of a scale to measure infant feeding attitudes and knowledge. Acta Paediatr 2008;97:1194-9.

42. Chen S, Binns CW, Liu Y, et al. Attitudes towards breastfeeding-the lowa Infant Feeding Attitude Scale in Chinese mothers living in China and Australia. Asia Pac J Clin Nutr 2013;22:266-9.

43. Dennis CL, Faux S. Development and psychometric testing of the breastfeeding self-efficacy scale. Res Nurs Health 1999;22:399-409.

44. Dai X, Dennis CL. Translation and validation of the breastfeeding self-efficacy scale into Chinese. J Midwifery Womens Health 2003;48:350-6. 